\title{
PARAMETER ESTIMATION IN NON-HOMOGENEOUS BOOLEAN MODELS: AN APPLICATION TO PLANT DEFENSE RESPONSE
}

\author{
María Ángeles Gallego ${ }^{1}$, María Victoria IbáÑeZ² And Amelia Simó凶,2 \\ ${ }^{1}$ Departament de Matemàtiques, Universitat Jaume I, Spain; ${ }^{2}$ Departament de Matemàtiques-IMAC, Universitat \\ Jaume I, Spain. \\ e-mail: mangeles.gallego.pitarch@gmail.com; mibanez@uji.es; simo@uji.es \\ (Received October 28, 2013; revised May 23, 2014; accepted September 23, 2014)
}

\begin{abstract}
Many medical and biological problems require to extract information from microscopical images. Boolean models have been extensively used to analyze binary images of random clumps in many scientific fields. In this paper, a particular type of Boolean model with an underlying non-stationary point process is considered. The intensity of the underlying point process is formulated as a fixed function of the distance to a region of interest. A method to estimate the parameters of this Boolean model is introduced, and its performance is checked in two different settings. Firstly, a comparative study with other existent methods is done using simulated data. Secondly, the method is applied to analyze the longleaf data set, which is a very popular data set in the context of point processes included in the $\mathrm{R}$ package spatstat. Obtained results show that the new method provides as accurate estimates as those obtained with more complex methods developed for the general case. Finally, to illustrate the application of this model and this method, a particular type of phytopathological images are analyzed. These images show callose depositions in leaves of Arabidopsis plants. The analysis of callose depositions, is very popular in the phytopathological literature to quantify activity of plant immunity.
\end{abstract}

Keywords: binary images, callose deposition, mixed volumes, non-homogeneous Boolean model, parameter estimation.

\section{INTRODUCTION}

In a wide variety of technological and scientific fields there are many practical situations in which researchers need to manage image data in order to obtain conclusions about a phenomenon of interest. Very often, these images are binary images showing the area covered by a given phenomenon in a certain region. A very appropriate probabilistic model for studying this kind of images, where the area covered by different events usually overlaps (random clumps), is the Boolean model (Molchanov, 1997; Chiu et al., 2013). This model is formed by placing random compact sets on the points of a Poisson point process and considering the union of these sets. Difficulties arise when certain grains overlap or remain completely covered by the others, thus making it impossible to perform direct measurements of the characteristics of the particles.

A formal definition of a Boolean model is as follows.

Definition 1 (Boolean model) Let $\Phi_{\lambda}=\left\{x_{1}, x_{2}, \ldots\right\}$ be a stationary Poisson point process in $\mathbb{R}^{d}$ of intensity $\lambda$. Let $\Xi_{1}, \Xi_{2}, \ldots$ be a sequence of independent identically distributed random compact sets in $\mathbb{R}^{d}$ that are independent of the Poisson process $\Phi_{\lambda}$ and satisfy $E v_{d}\left(\Xi_{0} \oplus \check{K}\right)<+\infty$ for all compacts $K \subset \mathbb{R}^{d}$, where
$\Xi_{0}$ is a random compact set of the same distribution as $\Xi_{n}, \check{K}=\{-x: x \in K\}$ and $v_{d}$ is the $d$-dimensional Lebesgue measure. The Boolean model $\Xi$ is then defined as:

$$
\Xi=\bigcup_{i}\left(x_{i}+\Xi_{i}\right) .
$$

The points $x_{i}$ are called germs, the sets $\Xi_{i}$ are known as grains and the random set $\Xi_{0}$ is said to be the typical grain of the Boolean model. The value of parameter $\lambda$ is said to be the intensity of the Boolean model.

If the grains have an isotropic distribution (distribution that is invariant under rotations about the origin), then the Boolean model is also isotropic in distribution.

A more in-depth study of this model can be found in Serra (1982), Ayala (1988), Cressie (1993), Molchanov (1997) and Chiu et al. (2013). Applications of Boolean models to real images have been worked by Lyman (1972), Margalef (1974), Serra (1982), Plaza (1991) and Chiu et al. (2013), among others.

The assumptions of stationarity and isotropy in Def. 1 facilitate the estimation of the parameters of the Boolean model. However, the hypothesis of spatial homogeneity frequently fails when real data sets are analyzed. Methods to estimate parameters of 
non-homogeneous Boolean models have been studied by Molchanov and Chiu (2000) and by Schmitt (1996). Non-homogeneous Boolean models have been used for the functional modeling of graded materials (Quintanilla and Torquato, 1997; Hahn et al., 1999), distributions of galaxies (Bond et al., 1995) and complex fluids (Brodatzki and Mecke, 2001).

Our aim in this paper is to propose an alternative statistical method to estimate the parameters of interest of a particular type of non-homogeneous Boolean model. This particular model can be applied to a great variety of practical situations. As an example, we will show its application to a phytopathological problem. Our method is similar in some respects to that of Berman and Turner (1992) for inhomogeneous point processes.

In this application, we will work with microscopic images obtained in the study of callose deposition in leaves of Arabidopsis plants (Luna et al., 2011). The study of callose deposition is a very popular method in the phytopathological literature to quantify plant immune system activity. Callose deposition in a leaf form random clumps, which are more densely distributed near the nerves of the leaf. So in this case, we can consider this deposition as a realization of a non-homogeneous Boolean model where the intensity of the Poisson point process is modeled as a known function of the distance from each point to the closest leaf nerve. As a result, a particular nonhomogeneous Boolean model can be assumed. As an alternative to the method proposed by Schmitt (1996) and Molchanov and Chiu (2000), we propose, for this model, a parameter estimation method based on a least squares fit procedure applied to the area fraction and to the density of the boundary length functions.

The rest of the paper is organized as follows: first the definition of non-homogeneous Boolean model will be introduced. A particular case of this model will be detailed in the following section. Then the parameter estimation procedures proposed by Molchanov and Schmitt will be explained, together with a new estimation procedure that will be proposed. Next a simulation study will be carried out to test the performance of the different parameter estimation procedures. In the subsequent section the proposed method is applied to a very popular data set. This data set is included in the $\mathrm{R}$ package spatstat and provides the locations and diameters of adult longleaf pines in a region. In this data set the germ process is known, so we can compare our results with those obtained using point process methods. Then this method will be applied to analyze microscopic images obtained in the study of callose deposition in plant leaves (Luna et al., 2011). Finally, conclusions will be stated.

\section{NON-HOMOGENEOUS BOOLEAN MODEL}

The assumption of stationarity in the definition of the Boolean model (Def. 1), means that the phenomenon being studied spreads over the plane in a homogeneous way. As has been stated previously, in practice this hypothesis usually fails, but it is still assumed as it facilitates the parameter estimation process.

In many real situations, where this hypothesis is not acceptable at all, a suitable model is the nonhomogeneous Boolean model. A non-homogeneous Boolean model (Molchanov, 1997; Chiu et al., 2013) is a Boolean model whose germ process is a nonhomogeneous Poisson point process that is obtained substituting the constant intensity of the homogeneous Poisson process by a general intensity measure $\Lambda(B)$, for $B \subset \mathbb{R}^{d}$. Usually $\Lambda(B)=\int_{B} \lambda(x) d x$. The density $\lambda(x)$, that is, finite and non-negative, is called the intensity function of the general Poisson point process. The formal definition of a non-homogeneous Boolean model is as follows.

\section{Definition 2 (Non-homogeneous Boolean model)}

Let $\Phi_{\lambda}=\left\{x_{1}, x_{2}, \ldots\right\}$ be a non-homogeneous Poisson process in $\mathbb{R}^{d}$ with intensity function $\lambda(x)$. Let $\Xi_{1}, \Xi_{2}, \ldots$ be a sequence of independent identically distributed random compact sets in $\mathbb{R}^{d}$ that are independent of the Poisson process $\Phi_{\lambda}$ and satisfy $E v_{d}\left(\Xi_{0} \oplus \check{K}\right)<+\infty$ for all compacts $K$, where $\Xi_{0}$ is a random compact set of the same distribution as $\Xi_{n}$, and $v_{d}$ is the d-dimensional Lebesgue measure. The non-homogeneous Boolean model (NHBM) $\Xi$ is $\Xi=\bigcup_{i}\left(x_{i}+\Xi_{i}\right)$.

The points $x_{i}$ are called germs, the sets $\Xi_{i}$ are known as grains and the random set $\Xi_{0}$ is referred to as the typical grain. A non-homogeneous Boolean model is characterized by its intensity function and the probability distribution of its typical grain. From now on, we will continue working on $\mathbb{R}^{2}$ i.e., fixing $d=2$.

Given a NHBM (Def. 2), its probability distribution is characterized by its capacity functional, which, for each compact set $K$, is defined as:

$$
\begin{aligned}
T_{\Xi}(K) & =P(\Xi \bigcap K \neq \emptyset) \\
& =1-\exp \left[-E\left\{\Lambda\left(K \oplus \check{\Xi}_{0}\right)\right\}\right],
\end{aligned}
$$

where $\check{\Xi}_{0}=\left\{-x: x \in \Xi_{0}\right\}$. 
The capacity functional for $K=\{x\}, p(x)=$ $T_{\Xi}(\{x\})$ is defined as the volume fraction function, in general, or as the area fraction function in the particular case of $d=2$. Therefore, by Eq. 2,

$$
p(x)=P(x \in \Xi)=1-\exp \left[-E\left\{\Lambda\left(x \oplus \check{\Xi}_{0}\right)\right\}\right],
$$

and by Fubini's theorem:

$$
p(x)=1-\exp \left[-E\left\{\int_{\check{\Xi}} \lambda(x-y) \mathrm{d} y\right\}\right] .
$$

The area fraction function is a particular case of the extended intrinsic volume densities $\bar{V}_{j}(\Xi ; x)$ $j=0,1,2$ of the Boolean model $\Xi . p(x)=\bar{V}_{2}(\Xi ; x)$. Another interesting extended intrinsic volume density is $\bar{V}_{1}(\Xi ; x)$ the density function of the boundary length, denoted by $L_{A}(x)$. The density of the boundary length of a NHMB at point $x$ is (Weil, 2001):

$$
L_{A}(x)=(1-p(x)) E\left\{\int_{\mathbb{R}^{2}} \lambda(x-y) \Phi_{1}(\Xi, \mathrm{d} y)\right\},
$$

where $\Phi_{1}(\Xi, d y)$ denotes the generalized curvature measure of $\Xi$ (Schneider and Weil, 2008). $2 \Phi_{1}(\Xi, B)$ is defined as the length of $\partial \Xi \bigcap B$, where $\partial \Xi$ denotes the boundary of $\Xi$ and $B \subset \mathbb{R}^{2}$ is a Borel set.

Non-homogeneous Boolean models with spherical grains in $\mathbb{R}^{2}$ and $\mathbb{R}^{3}$ have interesting applications in statistical physics, in particular for continuous percolation problems.

Estimators of the area fraction function and the density of the boundary length can be found in Molchanov (1997). We have to note that the measurement of the density of the boundary length is not very easy to implement on the digitized computer.

\section{A PARTICULAR CASE OF NON- HOMOGENEOUS BOOLEAN MODEL}

Departure from the homogeneity hypothesis can be due to a large number of different causes. In particular, in this paper we are going to focus our interest on one of the most common causes in practice: the case where the phenomenon of interest is spread over the plane non-homogeneously, depending on the distance to a certain focus or region of interest, ROI. Examples include proliferation of houses and/or industries close to main roads in a country; plants affected by a virus around the outbreak of the disease; radioactivity levels or cases of illness around a power plant, a nuclear plant or particular industries; destruction around the epicenter of an earthquake, and so on. In particular, in the example that we will analyze later, we will work with microscopic images of leaves, where callose deposition is non-homogeneously distributed across their surface, showing an intensity of events that depends on the proximity to the nerves of the leaves.

In order to take advantage of prior knowledge on the characteristics of the non-homogeneity, we suggest considering a particular NHBM with:

- A parametric intensity function $\lambda(x)=$ $f\left(D_{R O I}(x) ; a\right)$, where $f$ is a known function depending on $a=\left(a_{1}, \ldots, a_{p}\right)$, the parameters to estimate, $D_{R O I}(x)$ is the distance from point $x$ to the region of interest, $R O I$, and $f\left(D_{R O I}(x) ; a\right) \geq 0$ $\forall x \in \mathbb{R}^{2}$.

- A parametric grain distribution. We assume that the typical grain is a ball with a random radius whose probability distribution is known except for some parameters. i.e., $\Xi_{0}=B(0, r)$, where $B(0, r)$ denotes the ball centered in the origin and radius $r$ and $r$ has the probability density $g(r ; \theta)$, with $\theta=\left(\theta_{1}, \ldots, \theta_{k}\right)$.

Under these conditions, the area fraction function (Eq. 3) and the density of the boundary length (Eq. 4) become (Weil, 2001):

$$
\begin{aligned}
& p(x)=1- \\
& \exp \left[-\iint_{B(x, r)} f\left(D_{R O I}(y) ; a\right) g(r ; \theta) \mathrm{d} y \mathrm{~d} r\right],
\end{aligned}
$$

$$
\begin{aligned}
L_{A}(x)=(1-p(x)) . \\
\quad\left(\int_{\mathbb{R}^{2}} f\left(D_{R O I}(x-y) ; a\right) g(\|y\| ; \theta) \mathrm{d} y\right) .
\end{aligned}
$$

As an illustration, Fig. 1 shows realizations of a NHBM where it is assumed that the intensity of the underlying Poisson point process depends on the distance to a single point (Fig. 1a); two points (Fig. 1b); a line (Fig. 1c) a pair of lines (Fig. 1d). In all these realizations we assume a fixed value for the radius, $r=5$, and an exponential decay for the intensity function. In particular, it is assumed that $\lambda(x)=$ $0.01 \exp \left\{-0.05 D_{R O I}(x)\right\}$. Fig. 2 shows realizations of a NHBM with the same intensity functions as those seen in Fig. 1, but assuming a uniform probability distribution in $[0,10]$ for the radius. 


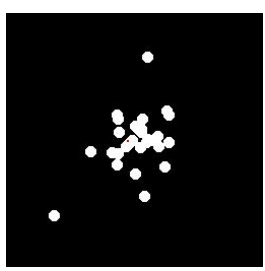

(a)

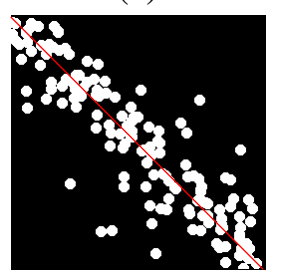

(c)

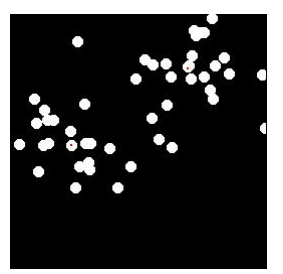

(b)

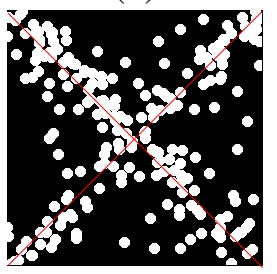

(d)
Fig. 1. Realizations of an NHBM with a fixed radius and intensity function depending on the distance to $(a)$ a single point (marked in red), (b) a pair of points(in red), (c) a single line (in red), (d) a pair of lines (in red).

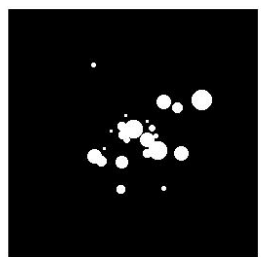

(a)

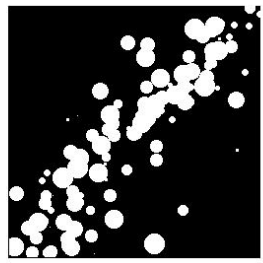

(c)

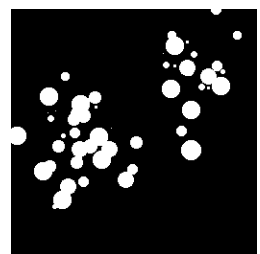

(b)

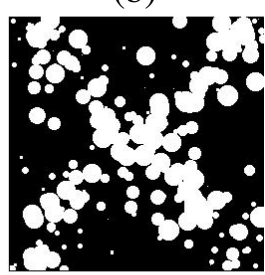

(d)
Fig. 2. Realizations of an NHBM with a uniform radius distribution and intensity function depending on the distance to (a) a single point, (b) a couple of points, (c) a single line, (d) a pair of lines.

\section{PARAMETER ESTIMATION IN NON-HOMOGENEOUS BOOLEAN MODELS}

Molchanov and Chiu (2000) and Schmitt (1996) developed different methods to estimate the parameters of both homogeneous and nonhomogeneous Boolean models. In this section, we will review the methods proposed for NHBM, and we will also propose an alternative estimation method for the particular case of NHBM introduced in the previous.

\section{MOLCHANOV METHOD}

Let $\Xi$ be a non-stationary Boolean model, with germ process $\Phi_{\lambda}$ and intensity function $\lambda(x)$, and let the typical grain $\Xi_{0}$, be a closed convex set.

Let us fix a direction $u$ in $\mathbb{R}^{2}$ and define the tangent point of each grain $\Xi_{i}$ as the lexicographical minimum among all points at which a hyperplane orthogonal to $u$ and moving in the direction of $u$ first touches $\Xi_{i}$ (Molchanov and Stoyan, 1994). Then, the observable tangent points form a point process, $\Psi$, of intensity function $\mu(x)$ (Molchanov and Chiu, 2000), and it is proved that if the area fraction function of the original Boolean model $p(x)$ is known or can be estimated, then:

$$
\lambda(x)=\mu(x) /\{1-p(x)\} .
$$

As $\Psi$ is non-stationary, the density of its intensity measure, $\mu(x)$, can be estimated by kernel methods (Bowman and Azzalini, 1997). If $k$ is a kernel and $h$ is a bandwidth, then $\mu$ is estimated by

$$
\hat{\mu}(x)=\sum_{x_{i} \in \Psi} k\left\{\left(x-x_{i}\right) / h\right\} .
$$

If necessary, the area fraction function can also be estimated by a nonparametric regression estimator as:

$$
\hat{p}(x)=\frac{\int_{\Xi \cap W} k_{1}\left\{(y-x) / h_{1}\right\} \mathrm{d} y}{\int_{W} k_{1}\left\{(y-x) / h_{1}\right\} \mathrm{d} y},
$$

where $W \subset \mathbb{R}^{2}$ is an observation window; $k_{1}$ is another kernel and $h_{1}$ is another bandwidth, which may be the same as the kernel $k$ and the bandwidth $h$ used to estimate $\hat{\mu}(x)$, or may differ.

Another version of this method (Molchanov and Chiu, 2000) also allows us to estimate the parameters of the probability distribution of the typical grain. We have not used it due to its excessive computational cost.

\section{SCHMITT METHOD}

This method, suggested by Schmitt (1996) for the non-stationary case, can be applied to Boolean models with primary grains almost surely bounded by a square of edge length $r$.

It makes it possible to estimate the mean of the non-stationary intensity inside a square of arbitrary size $\varepsilon$, as:

$$
\int_{[0, \varepsilon]^{2}} \lambda(x) \mathrm{d} x=\log \frac{\left(1-T_{\Xi}(G)\right)\left(1-T_{\Xi}(G \cup K \cup L)\right)}{\left(1-T_{\Xi}(G \cup L)\right)\left(1-T_{\Xi}(G \cup K)\right)},
$$

$$
\forall \varepsilon>0 \text {, }
$$


where sets $G, L$ and $K$ must be chosen in a special way. In his paper, Schmitt proposed to definee the sets as shown in Fig. 3.

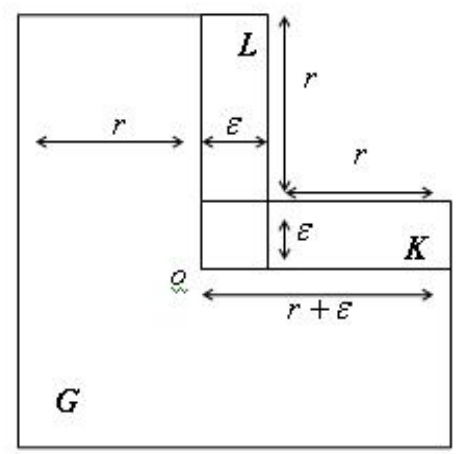

Fig. 3. The sets used in the definition of Schmitt's estimator.

\section{LEAST SQUARES ON INTRINSIC VOLUME DENSITIES (LSIVD) METHOD}

Molchanov et al. and Schmitt, propose methods to estimate the parameters of the intensity function of a NHBM, in general, without assuming any knowledge about the causes of non-homogeneity. But if we could have any knowledge about theses causes, i.e., if we could have additional information about the characteristics of the intensity function, it would seem more appropriate to use such information in the estimation process in order to reduce the computational complexity and obtain estimates as accurate as those obtained by other more general methods. This is the main aim of the method proposed in this section, to use prior knowledge about the functional form of $\lambda(x)$ in order to estimate its parameters using a conceptually less demanding algorithm.

The outline of the algorithm is as follows:

1. To estimate the area fraction function, $\widehat{p}(x)$, and the density of the boundary length $\widehat{L_{A}}(x)$, for a grid of $x$-values in $W$ by using a kernel estimator, as in Eq. 8.

2. Following Eqs. 5, 6, use numerical methods to approximate:

$$
\begin{aligned}
& Q_{1}(x, a, \theta)=\iint_{B(x, r)} f\left(D_{R O I}(y) ; a\right) g(r ; \theta) \mathrm{d} y \mathrm{~d} r, \\
& Q_{2}(x, a, \theta)=\int_{\mathbb{R}^{2}} f\left(D_{R O I}(x-y) ; a\right) g(\|y\| ; \theta) \mathrm{d} y,
\end{aligned}
$$

which will be possible if $f\left(D_{R O I}(x) ; a\right)$ has a simple functional form.
3. To estimate the parameters $a=\left(a_{1}, \ldots, a_{p}\right)$ and $\theta=\left(\theta_{1}, \ldots, \theta_{k}\right)$, by least squares, minimizing

$$
\begin{gathered}
Q(a, \theta)=\sum_{x_{i} \in W}[ \\
\frac{1}{(n-1) S_{\hat{Q}_{1}}^{2}}\left(\ln \left(1-\widehat{p}\left(x_{i}\right)\right)-\left(-Q_{1}\left(x_{i}, a, \theta\right)\right)\right)^{2}+ \\
\left.+\frac{1}{(n-1) S_{\hat{Q}_{2}}^{2}}\left(\frac{\widehat{L}_{A}\left(x_{i}\right)}{1-\widehat{p}\left(x_{i}\right)}-Q_{2}\left(x_{i}, a, \theta\right)\right)^{2}\right],
\end{gathered}
$$

where $\left\{x_{i}: x_{i} \in W, i=1, \ldots, n\right\}$ is a digital grid, $\widehat{Q}_{1}(x)=\ln (1-\widehat{p}(x)), \widehat{Q}_{2}(x)=\widehat{L}_{A}(x) /(1-\widehat{p}(x))$ and $S_{\hat{Q}_{1}}^{2}, S_{\hat{Q}_{2}}^{2}$ are their respective sample variances.

In our applications, we have used well-known numerical methods to approximate the integrals (Eq. 10) and to minimize the function given in Eq. 11. In particular, the trapezoidal rule has been used to approximate the integrals (Eq. 10), and the fmincon function of the Matlab optimization toolbox has been used to solve the optimization problem (Eq. 11). The purpose of fmincon is to find a constrained minimum of a scalar function of several variables starting at an initial estimate. It uses a sequential quadratic programming method, solving a quadratic programming $(\mathrm{QP})$ sub-problem at each iteration. At each iteration, an estimate of the Hessian of the Lagrangian is updated using the Broyden-Fletcher-Goldfarb-Shanno (BFGS) formula (Fletcher and Powell, 1963; Goldfarb, 1970); a line search is performed using a merit function similar to that proposed by Han (1977); Powell (1978a;b), and the QP subproblem is solved using an active set strategy similar to that described in (Gill et al., 1981). Researchers more expert than us in this field, will surely find numerical methods that provide better results, but our aim has simply been to show the method.

At this point we would like to point out that we are just concerned with a particular type of $\lambda(x)$ functions, whose functional form has been stated before. This type of intensity function is quite common in real applications, although functions $f$ and/or $g$ could be unknown. If $f$ and/or $g$ were unknown a reasonable approximation could be considered; for example, $f$ could be modeled as a linear or exponential function and $g$ could be considered as a normal or uniform density function.

In some cases, when the analytical expression of functions $Q_{1}$ and $Q_{2}$ are known, the numerical integration step of the algorithm can be omitted. Even in some cases, the optimization can also be obtained analytically. Let us see an example. 
Example 1 Let us consider a particular case of $\operatorname{NHBM}$ (Def. 2) with $\lambda(x)=a_{1}+a_{2} D_{R O I}(x)$, being $a=\left(a_{1}, a_{2}\right)$ constants to estimate. The radius of the ball, $r$, is considered fixed but unknown, and the region of interest is a line. In this case:

$$
\begin{aligned}
& Q_{1}(x, a, r)=\int_{B(x, r)} a_{1}+a_{2} D_{R O I}(y) \mathrm{d} y, \\
& Q_{2}(x, a, r)=\int_{0}^{2 \pi} r\left(a_{1}\right. \\
& \left.+a_{2} D_{R O I}(x-(r \cos (\theta), r \sin (\theta)))\right) \mathrm{d} \theta,
\end{aligned}
$$

and using the mean value theorem in both equations:

$$
\begin{aligned}
& Q_{1}(x, a, r)=\pi r^{2}\left(a_{1}+a_{2} D_{R O I}(x)\right), \\
& Q_{2}(x, a, r)=2 \pi r\left(a_{1}+a_{2} D_{R O I}(x)\right) .
\end{aligned}
$$

Thus it is not necessary to use any numerical optimization algorithm to obtain $\hat{a_{1}}, \hat{a_{2}}$ and $\hat{r}$. Using elemental calculus, similar to those used to obtain de minimum least squared line, the values of the parameters to minimize Eq. 11 will be:

$$
\hat{r}=\Delta+\sqrt{\Delta^{2}+4 \frac{S_{\hat{Q}_{1}}^{2}}{S_{\hat{Q}_{2}}^{2}}},
$$

$\hat{a}_{1}=\frac{\hat{r} S_{D \hat{Q}_{1}} S_{\hat{Q}_{2}}^{2}+2 S_{D \hat{Q}_{2}} S_{\hat{Q}_{1}}^{2}}{\pi \widehat{r}\left(\hat{r}^{2} S_{\hat{Q}_{2}}^{2}+4 S_{\hat{Q}_{1}}^{2}\right) S_{D}^{2}}$,

$\hat{a}_{2}=\frac{S_{D}^{2}\left(\hat{r} \widehat{\widehat{Q}}_{1} S_{\hat{Q}_{2}}^{2}+2{\overline{Q_{2}}}_{2}\right) S_{\hat{Q}_{1}}^{2}-\bar{D}\left(\hat{r} S_{D \hat{Q}_{1}} S_{\hat{Q}_{2}}^{2}+2 S_{D \hat{Q}_{2}} S_{\hat{Q}_{1}}^{2}\right)}{\pi \hat{r}\left(\hat{r}^{2} S_{\hat{Q}_{2}}^{2}+4 S_{\hat{Q}_{1}}^{2}\right) S_{D}^{2}}$.

Being $\overline{\widehat{Q}}_{1}$ and $\overline{\widehat{Q}}_{2}$ the means of $\ln (1-\hat{p}(x))$ and $\hat{L}_{A}(x) /(1-\hat{p}(x))$ respectively; $\bar{D}$, and $S_{D}^{2}$, the mean and the variance of $D_{R O I}(x) ; S_{f g}=\left(\sum_{i}\left(f\left(x_{i}\right)-\right.\right.$ $\left.\bar{f})\left(g\left(x_{i}\right)-\bar{g}\right)\right) / n$ and

$\Delta=\frac{S_{D \hat{Q}_{1}}^{2} S_{\hat{Q}_{2}}^{2}-S_{D \hat{Q}_{2}}^{2} S_{\hat{Q}_{1}}^{2}+n S_{D}^{2}\left({\overline{Q_{1}}}_{1}^{2} S_{\hat{Q}_{2}}^{2}-\overline{\widehat{Q}}_{2}^{2} S_{\hat{Q}_{1}}^{2}\right)}{S_{\hat{Q}_{2}}^{2}\left(S_{D \hat{Q}_{1}} S_{D \hat{Q}_{2}}+n S_{D}^{2} \widehat{\widehat{Q}}_{1}{\widehat{Q_{2}}}_{2}\right)}$.

\section{SIMULATION STUDY}

In this section, a simulation study is carried out to test the performance of the three estimation procedures explained previously.

Three different experiments are performed. For each one, 20 realizations of the particular nonhomogeneous Boolean model introduced before are simulated on a $512 \times 512$ window. Regarding the model:
- The typical grain is assumed to be a ball, i.e., $\Xi_{0}=B(0, r)$.

- Two different intensity functions are considered:

$$
\lambda(x)=a_{1}+a_{2} D_{R O I}(x)
$$

and

$$
\lambda(x)=a_{1} \exp \left\{a_{2} D_{R O I}(x)\right\}
$$

$a_{1}$ and $a_{2}$ being the parameters to estimate, and $D_{R O I}(x)$ the distance from point $x$ to the region of interest.

- Two types of regions of interest, will also be considered in each experiment: a line, and two different lines.

Fig. 1 shows realizations of some of these particular NHBMs assuming that $\lambda(x)=$ $0.01 \exp \left\{-0.05 D_{R O I}(x)\right\}$ and that $D_{R O I}(x)$ represents the distance to a line (Fig. 1 (c)) and to two crossing lines (Fig. 1 (d)).

Results obtained using Molchanov's, Schmitt's and LSIVD methods will be compared.

Regarding our method:

- An Epanechnikov kernel with a bandwidth of $h=$ 70 has been used to estimate the area fraction function and the density of the boundary length (Eq. 8).

- As stated below, the trapezoidal rule has been used to approximate $Q_{i}(x, a, \theta), i=1,2$ (Eq. 10), and the fmincon function of the Matlab optimization toolbox has been used to minimize the function stated in Eq. 11.

For smoothing purposes, in the method of Molchanov and Chiu (2000), the Epanechnikov kernel with a bandwidth $h$ of 70 has also been used to estimate the area fraction function (Eq. 8).

To perform Schmitt's method it is necessary to choose a value for the tuning parameter $\varepsilon$, which is related to the radius of the grain process. As the distribution of the radius is known in our simulations, we will detail in each case, the $\varepsilon$ value used.

All the algorithms have been implemented in Matlab ${ }^{1}$.

\footnotetext{
${ }^{1}$ MATLAB is a trademark of The MathWorks Inc.
} 


\section{FIRST EXPERIMENT}

In this first experiment we obtain 20 simulations of a non-homogeneous Boolean model with intensity function $\lambda(x)=a+b D(x)$. Two different combinations of $a, b$ and $r$ values are considered for image simulation: $a=1 \mathrm{e}-5, b=2 \mathrm{e}-5$ (where e-5 denotes $\times 10^{-5}$ ) and two different $r$-values: $r=5$ and $r=10$. To choose these values, it should be taken into account that not every combination is valid. There will be combinations that could lead to images without any interest, for being either completely covered, or almost empty. For this reason they have been chosen ad hoc, after visualizing the simulated images. To perform Schmitt's method, $\varepsilon=r+2$ has been used.

Once obtained the images, parameters $a, b$ and $r$ are estimated from them with the different methods exposed in the paper. Results of this first experiment can be found in Table 1 .

\section{SECOND EXPERIMENT}

Let's consider an exponential expression for the intensity function, i.e., $\lambda(x)=a \exp \left\{-b D_{R O I}(x)\right\}$ with $a$ and $b$ being the constants to estimate. The radius of the ball, $r$, is considered fixed but unknown.

Once again, parameters are chosen following the criteria explained in the first experiment. To perform Schmitt's method, $\varepsilon=r+2$ has been used.

The results are shown in Table 2.

\section{THIRD EXPERIMENT}

Let's consider an exponential expression for the intensity function, i.e., $\lambda(x)=a \exp \left\{-b D_{R O I}(x)\right\}$, with $a$ and $b$ being the constants to estimate, and let $r$ follow a uniform distribution on $[0, R]$.

Once again, parameters are chosen in the same way as in the previous cases. To perform Schmitt's method, $\varepsilon=R+2$ has been used.

The results are shown in Table 3.

\section{COMMENTS ON THE RESULTS}

The main advantage of LSIVD method is its ability to estimate $r$ (or $R$ ), in addition to the parameters of the intensity function, $a$ and $b$, with a very simple algorithm. As can be seen in Tables 1,2 and 3, the three methods provide quite similar estimations, although subtle differences can be found between them. Molchanov's method is the one that usually provides less accurate estimations, while LSIVD method is the one that usually presents the lowest variability in the estimations obtained. It is Schmitt's method that achieves the most accurate estimations in the highest number of cases.

It must also be noted that parameter $r$ (respectively $R$ ), is the parameter most efficiently estimated, although it tends to be slightly overestimated.

Note that the performance of LSIVD method is usually somewhat worse than Schmitt's, but Schmitt's method cannot estimate the value of $r$. Additionally, in real applications the value of the tuning parameter $\varepsilon$ is unknown and its estimation could affect the performance of the method. There is a modified version of Molchanov's algorithm that makes it possible to estimate $r$ together with the parameters of the intensity function, however, Molchanov provides a bit worse estimations than LSIVD method.

When analyzing the results, the difficulty of working with digital images should be kept in mind. For example, our circles are circles in a digital grid and it is therefore difficult to obtain a precise measure of their boundary length or their area. In our opinion, this may be causing the bias in the observed estimates. Our method could be improved with better digital measurements for the length of the curves and the area and with more accurate numerical methods for approaching the integrals and the optimization. In the present study, the Matlab function edge has been used to get the boundaries, and the number of pixels of these boundaries has been used to measure the lengths. The area has been also measured counting the number of pixels.

\section{ANALYZING LONGLEAF PINES DATA SET}

spatstat (Baddeley and Turner, 2005) is a popular R-package for analyzing bidimensional point patterns, which includes some standard point pattern data sets. Among these standard data sets we can find the Longleaf Pines data set (Platt et al., 1988; Rathbun and Cressie, 1994), which is available as longleaf. The longleaf data set provides the location and diameters at breast height (dbh, a convenient measure of their size) of 584 Longleaf Pine (Pinus palustris) trees in a $200 \mathrm{~m} \times 200 \mathrm{~m}$ region in southern Georgia (USA). This data set represents a marked point pattern and it has been largely analyzed as an example of spatially inhomogeneous point pattern (Baddeley et al., 2000; Perry and Enright, 2006). Like Baddeley et al. (2000) and Perry and Enright (2006), we have considered only 'adult' trees, which are conventionally defined as those with a dbh greater than or equal to $30 \mathrm{~cm}$ (Platt et al., 1988). 
Table 1. Mean and standard deviation of the estimates of the parameters $a, b$, and $r$ of the non-homogeneous Boolean model set in the first experiment.

\begin{tabular}{|c|c|c|c|c|c|c|c|}
\hline \multirow{2}{*}{ True value } & \multirow[b]{2}{*}{ ROI } & \multicolumn{2}{|c|}{ Schmitt method } & \multicolumn{2}{|c|}{ Molchanov method } & \multicolumn{2}{|c|}{ LSIVD Method } \\
\hline & & mean & sd & mean & sd & mean & sd \\
\hline$a=1.00 \mathrm{e}-5$ & & $68.00 e-5$ & $77.00 \mathrm{e}-5$ & $16.00 \mathrm{e}-5$ & $13.00 \mathrm{e}-5$ & $1.00 \mathrm{e}-5$ & $5.73 e-42$ \\
\hline$b=2.00 \mathrm{e}-5$ & Line & $421.00 \mathrm{e}-5$ & $1676.00 \mathrm{e}-5$ & $0.000002 \mathrm{e}-5$ & $0.0000009 \mathrm{e}-5$ & $2.10 e-5$ & $1.14 \mathrm{e}-41$ \\
\hline$r=5.00$ & & & & & & 5.10 & 0.32 \\
\hline$a=1.00 \mathrm{e}-5$ & & $15.00 \mathrm{e}-5$ & $93 e-5$ & $11.00 \mathrm{e}-5$ & $12.00 \mathrm{e}-5$ & $1.02 \mathrm{e}-5$ & $0.00000000489 e-5$ \\
\hline$b=4.00 \mathrm{e}-5$ & Two lines & $0.00006 e-5$ & $0.006 e-5$ & $1.80 e-5$ & $0.59 e-6$ & $4.11 \mathrm{e}-5$ & $0.0000000783 e-5$ \\
\hline$r=5.00$ & & & & & & 5 & 0 \\
\hline$a=1.00 \mathrm{e}-5$ & & $0.00003 e-5$ & $0.00002 \mathrm{e}-5$ & $45.00 \mathrm{e}-5$ & $19.00 \mathrm{e}-5$ & $8.60 e-5$ & $9.80 \mathrm{e}-5$ \\
\hline$b=2.00 \mathrm{e}-5$ & Line & $6.60 e-5$ & $0.30 e-5$ & $0.072 \mathrm{e}-5$ & $0.23 e-5$ & $2.00 e-5$ & $0.08 e-5$ \\
\hline$r=10.00$ & & & & & & 10.10 & 0.32 \\
\hline$a=1.00 \mathrm{e}-5$ & & $15.00 \mathrm{e}-5$ & $12.00 \mathrm{e}-5$ & $52.00 \mathrm{e}-5$ & $95.00 \mathrm{e}-5$ & $0.830 \mathrm{e}-5$ & $0.000000907 e-5$ \\
\hline$b=4.00 \mathrm{e}-5$ & Two lines & $0.83 e-5$ & $1.90 e-5$ & $1.10 \mathrm{e}-5$ & $0.19 e-5$ & $4.00 e-5$ & $0.002 \mathrm{e}-5$ \\
\hline$r=10.00$ & & & & & & 10 & 0 \\
\hline
\end{tabular}

Table 2. Mean and standard deviation of the estimates of the parameters $a$, $b$, and $r$ of the non-homogeneous Boolean model set in the second experiment.

\begin{tabular}{|c|c|c|c|c|c|c|c|}
\hline & & \multicolumn{2}{|c|}{ Schmitt method } & \multicolumn{2}{|c|}{ Molchanov method } & \multicolumn{2}{|c|}{ LSIVD Method } \\
\hline True value & ROI & mean & sd & mean & sd & mean & sd \\
\hline$a=1.00 \mathrm{e}-2$ & & $1.02 \mathrm{e}-2$ & $1.55 \mathrm{e}-2$ & $0.40 \mathrm{e}-2$ & $0.05 \mathrm{e}-2$ & $0.53 \mathrm{e}-2$ & $0.04 \mathrm{e}-2$ \\
\hline$b=5.00 \mathrm{e}-2$ & Line & $7.13 \mathrm{e}-2$ & $15.21 \mathrm{e}-2$ & $2.61 \mathrm{e}-2$ & $0.22 \mathrm{e}-2$ & $2.35 \mathrm{e}-2$ & $0.14 \mathrm{e}-2$ \\
\hline$r=5.00$ & & & & & & 5.80 & 0.63 \\
\hline$a=1.00 \mathrm{e}-2$ & & $1.72 \mathrm{e}-2$ & $2.07 e-2$ & $0.36 \mathrm{e}-2$ & $0.03 \mathrm{e}-2$ & $0.54 \mathrm{e}-2$ & $0.02 \mathrm{e}-2$ \\
\hline$b=5.00 \mathrm{e}-2$ & Two lines & $8.69 e-2$ & $18.41 \mathrm{e}-2$ & $1.98 \mathrm{e}-2$ & $0.21 \mathrm{e}-2$ & $2.18 \mathrm{e}-2$ & $0.05 \mathrm{e}-2$ \\
\hline$r=5.00$ & & & & & & 5.80 & 0.63 \\
\hline$a=1.00 \mathrm{e}-2$ & & $1.05 \mathrm{e}-2$ & $1.10 \mathrm{e}-2$ & $0.25 \mathrm{e}-2$ & $0.07 e-2$ & $0.48 \mathrm{e}-2$ & $0.53 e-2$ \\
\hline$b=5.00 \mathrm{e}-2$ & Line & $6.43 e-2$ & $13.59 \mathrm{e}-2$ & $2.41 \mathrm{e}-2$ & $0.41 \mathrm{e}-2$ & $4.23 e-2$ & $1.24 \mathrm{e}-2$ \\
\hline$r=10.00$ & & & & & & 10.30 & 0.48 \\
\hline$a=1.00 \mathrm{e}-2$ & & $8.28 \mathrm{e}-2$ & $2.21 \mathrm{e}-2$ & $0.21 \mathrm{e}-2$ & $0.05 \mathrm{e}-2$ & $0.50 \mathrm{e}-2$ & $0.04 \mathrm{e}-2$ \\
\hline$b=5.00 \mathrm{e}-2$ & Two lines & $5.85 e-2$ & $1.69 \mathrm{e}-2$ & $1.60 \mathrm{e}-2$ & $0.47 \mathrm{e}-2$ & $4.74 \mathrm{e}-2$ & $0.83 e-2$ \\
\hline$r=10.00$ & & & & & & 10.10 & 0.31 \\
\hline
\end{tabular}

From this data set we are going to use our method to estimate the parameters of the NHBM formed by the germ process of the locations of the trees and grain process of balls with a radius proportional to their respective diameters. It should be noted that it is not frequent to know the locations of trees in a forest because this is an expensive and hard task. Let us imagine that just an aerial photograph of this area was available for the study, then our sample information would be just a digital image showing the area covered by the trees. Assuming that the diameter of the crown of the tree is proportional to its diameter at breast height, the information available usually in this kind of problem is a binary image like the one shown in Fig. 4b.

Looking at Fig. $4 \mathrm{~b}$ we can see that the area covered by trees is clearly growing from right to left in the region, so we can try to adjust the data to a non-homogeneous Boolean model assuming that the intensity at a point $x=\left(x_{1}, x_{2}\right)$, is a function of the distance from $x$ to the $x_{2}$-axis. An exponential function is assumed, i.e., $\lambda(x)=a \exp \{-b D(x)\}$. With respect to the radius distribution we are going to assume the most simple one, a uniform distribution in $[30, r]$. Therefore, we have three parameters to estimate: $a, b$ and $r$.

Applying the method explained in section 4.3, the estimates obtained are: $\hat{a}=0.003, \hat{b}=-0.0062$ and $\hat{r}=65$.

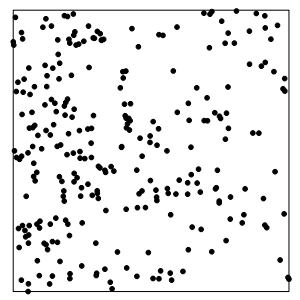

(a)

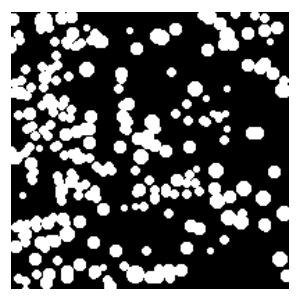

(b)
Fig. 4. (a) Position of 271 adult pine trees in a forest (longleaf dataset from the spatstat package). (b) Binary image showing the area covered by the pine trees assuming that the diameter of the crown of the trees is proportional to their diameter at breast height. 
Table 3. Mean and standard deviation of the estimates of parameters $a, b$, and $R$ of the non-homogeneous Boolean model set in the third experiment.

\begin{tabular}{|c|c|c|c|c|c|c|c|}
\hline \multirow{2}{*}{ True value } & \multirow[b]{2}{*}{ ROI } & \multicolumn{2}{|c|}{ Schmitt method } & \multicolumn{2}{|c|}{ Molchanov method } & \multicolumn{2}{|c|}{ LSIVD method } \\
\hline & & mean & $\mathrm{sd}$ & mean & sd & mean & sd \\
\hline$a=1.00 \mathrm{e}-2$ & & $0.47 e-2$ & $0.38 \mathrm{e}-2$ & $0.51 \mathrm{e}-2$ & $0.05 \mathrm{e}-2$ & $0.55 \mathrm{e}-2$ & $0.07 e-2$ \\
\hline$b=5.00 \mathrm{e}-2$ & Line & $3.02 \mathrm{e}-2$ & $1.20 \mathrm{e}-2$ & $2.67 \mathrm{e}-2$ & $0.17 \mathrm{e}-2$ & $2.30 \mathrm{e}-2$ & $0.08 \mathrm{e}-2$ \\
\hline$R=5$ & & & & & & 6.27 & 0.81 \\
\hline$a=1.00 \mathrm{e}-2$ & & $0.67 e-2$ & $0.51 \mathrm{e}-2$ & $0.50 \mathrm{e}-2$ & $0.04 \mathrm{e}-2$ & $0.57 e-2$ & $0.03 e-2$ \\
\hline$b=5.00 \mathrm{e}-2$ & Two lines & $5.52 \mathrm{e}-2$ & $13.41 \mathrm{e}-2$ & $2.13 \mathrm{e}-2$ & $0.16 \mathrm{e}-2$ & $2.16 \mathrm{e}-2$ & $0.05 e-2$ \\
\hline$R=5$ & & & & & & 6.19 & 0.22 \\
\hline$a=1.00 \mathrm{e}-2$ & & $1.13 \mathrm{e}-2$ & $1.64 \mathrm{e}-2$ & $0.41 \mathrm{e}-2$ & $0.06 \mathrm{e}-2$ & $0.36 \mathrm{e}-2$ & $0.08 \mathrm{e}-2$ \\
\hline$b=5.00 \mathrm{e}-2$ & Line & $7.39 \mathrm{e}-2$ & $11.32 \mathrm{e}-2$ & $2.66 \mathrm{e}-2$ & $0.25 \mathrm{e}-2$ & $2.37 \mathrm{e}-2$ & $0.08 \mathrm{e}-2$ \\
\hline$R=10$ & & & & & & 13.28 & 2.11 \\
\hline$a=1.00 \mathrm{e}-2$ & & $5.96 \mathrm{e}-2$ & $3.73 \mathrm{e}-2$ & $0.31 \mathrm{e}-2$ & $0.37 \mathrm{e}-2$ & $0.35 \mathrm{e}-2$ & $0.14 \mathrm{e}-2$ \\
\hline$b=5.00 \mathrm{e}-2$ & Two lines & $7.53 \mathrm{e}-2$ & $13.43 \mathrm{e}-2$ & $2.02 \mathrm{e}-2$ & $0.26 \mathrm{e}-2$ & $2.01 \mathrm{e}-2$ & $0.71 \mathrm{e}-2$ \\
\hline$R=10$ & & & & & & 11.33 & 4.43 \\
\hline
\end{tabular}

We are going to use this "artificial" example to illustrate the performance of our proposed method. In this case, the germ point process locations are known (see Fig. 4a) and this allows us to estimate their intensity function using the point processes methods implemented in the spatstat package. Therefore, we can compare it with the one obtained using our method applied to Fig. 4b. This comparison can be seen in Fig. 5. The results are quite promising taking into account the difference in the information available in each case. Fig. 6 shows a couple of simulations of the fitted model for the image in Fig. 4b.

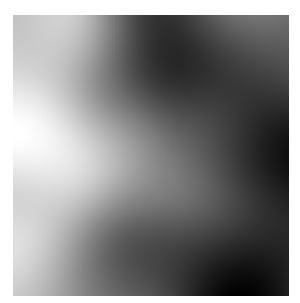

(a)

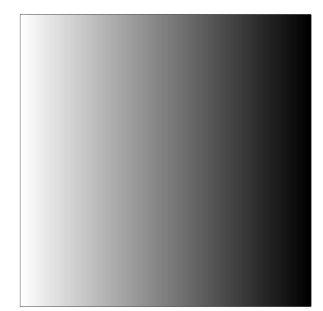

(b)
Fig. 5. (a) Estimated density function obtained using point processes methods applied to Fig. 4a,b estimated density function obtained using our method applied to Fig. $4 b$.
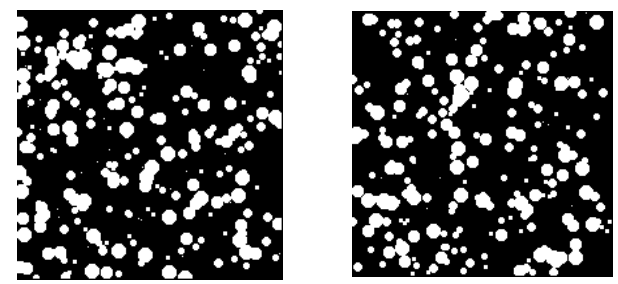

Fig. 6. Simulations of the non-homogeneous Boolean model fitted to the image of Fig. $4 b$.

\section{GOODNESS OF FIT TEST}

Once the parameters of the model have been estimated, it is necessary to check the adequacy of the model proposed, for modeling our data set. To this end, a Monte Carlo test of goodness of fit (Besag and Diggle, 1977; Diggle, 1983) is carried out.

We simulate 99 realizations of the model with the parameters estimated. In order to describe the realizations, $K_{\text {inhom, a generalization of the Ripley's }}$ $K$-function for second-order intensity-reweighted stationary germ-grain models has been chosen (Gallego et al., 2014):

$$
K_{\text {inhom }}(t)=\frac{1}{|W|} E\left(\int_{\Xi \cap W} \int_{\Xi \cap B(y, t)} \frac{1}{p(x) p(y)} \mathrm{d} y \mathrm{~d} x\right) .
$$

Let $\hat{K}_{\text {inhom }_{0}}$ and $\hat{K}_{\text {inhom }_{i}}(i=1, \cdots, 99)$ be the estimates of the $K_{\text {inhom-function from the real image }}$ and the simulations. Fig. 7 shows the function estimated from the real data, $\hat{K}_{\text {inhom }_{0}}$, and the lower and upper envelopes of $\hat{K}_{\text {inhom }_{i}}$ estimated from the simulations. This indicates a satisfactory fit because $\hat{K}_{\text {inhom }_{0}}$ lies between the two envelopes except for a few values.

Following Diggle (1983), we consider:

$$
\begin{array}{r}
D_{i}=\left(\int_{0}^{t_{0}}\left(\hat{K}_{\mathrm{inhom}_{i}}(t)-\bar{K}_{\mathrm{inhom}_{i}}(t)\right)^{2} \mathrm{~d} t\right)^{1 / 2}, \\
i=0, \ldots, 99,
\end{array}
$$

where $\bar{K}_{\text {inhom }_{i}}=\sum_{j \neq i} \hat{K}_{\text {inhom }_{j}} / 99$.

Let $r_{0}$ be the rank of $D_{0}$. For $r_{0}>50$ the Monte Carlo $p$-value is $2\left(100-r_{0}\right) / 100$. Meanwhile for $r_{0} \leq$ 50 it is $2\left(r_{0} / 100\right)$. The p-value obtained was 0.383 , so we can assume that the distribution of the pines is an non-homogeneous Boolean model with intensity function and the distribution of radios introduced above. 


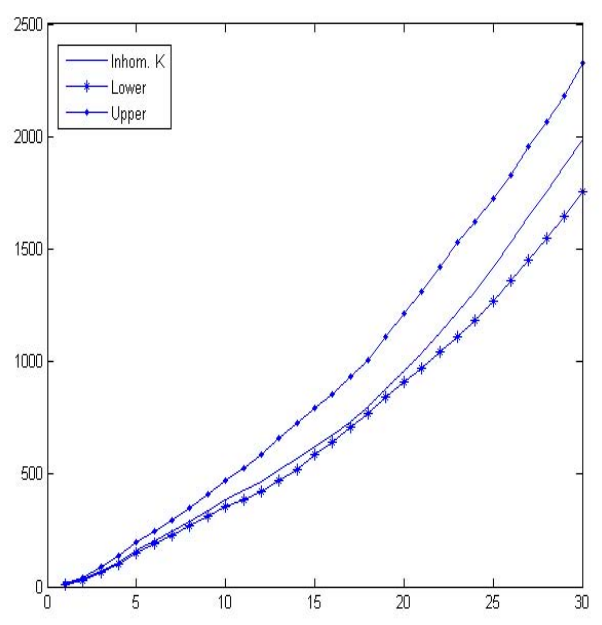

Fig. 7. Lower and upper envelopes of $\hat{K}_{\text {inhom }}$ estimates from the simulations and the estimated from the real data.

\section{APPLICATION}

In this section, we show how the NHBMs and our parameter estimation method can be applied to a phytopathological problem. As an illustration, we are going to analyze just one of the images obtained in an experimental study conducted by a group of researches from the Plant Physiology Section of Universitat Jaume I. The goal of their experimental study was to examine the robustness of callose deposition in response to different pathogenassociated molecular patterns (Luna et al., 2011). As a result of the experiment, they obtained epifluorescence microscopic images with a UV filter, of size $1536 \times$ 1920 pixels. As a preprocessing step, one of these colored images is selected and segmented into two binary images, one showing the nerves of the leaf and the other showing the callose deposition (Fig. 8). This second image shows small overlapping circular white spots. Mathematical morphological tools have been used to segment and convert the original image into binary images.

As can be seen in Fig. 8, callose deposition is mainly located close to the leaf nerves, presents a roughly circular shape and overlaps. That is why we propose to model their distribution with a nonhomogeneous Boolean model, assuming that the intensity at a point $x$, is a negative exponential function of the distance from $x$ to the closest nerve or to the edge of the leaf, i.e., $\lambda(x)=a \exp \{-b D(x)\}$, with $D(X)$ defined as the minimum between the Euclidean distance from a location $x$ to its nearest nerve and to the edge of the leaf. With this intensity function, there will only be two parameters to estimate: $a$ and $b$. If we additionally assume that the grains are balls of a fixed unknown radius $r$, a third parameter must also be estimated.

In the parameter estimation step, the algorithm previously detailed is followed. In order to estimate the area fraction (step 1) the Epanechnikov kernel is used with band bandwidth $h=80$. In step 3 , as in our simulation study, the trapezoidal rule is used to approximate the integral and the fmincon function of the Matlab optimization toolbox has been used to solve the optimization problem.

The results obtained were $\hat{a}=0.005, \hat{b}=1.5$ and $\hat{r}=2$ pixels.

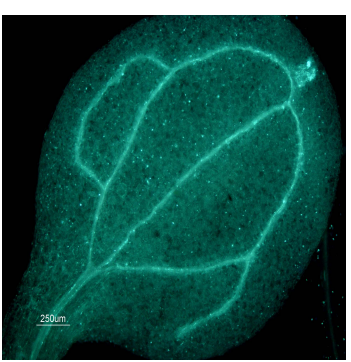

(a)

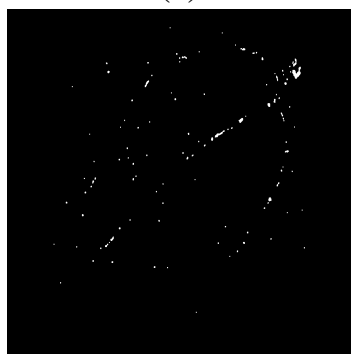

(c)

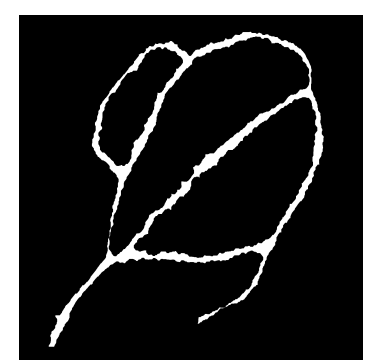

(b)

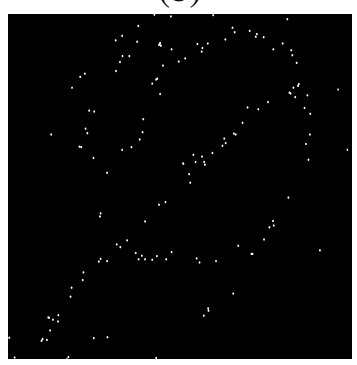

(d)
Fig. 8. (a) Original image. (b) Binary image showing the nerves of $(a)$. (c) Callose deposition image of $(a)$. (d) A simulation of the estimated model for the callose deposition image (c).

\section{GOODNESS OF FIT TEST}

Once we have estimated the parameters of the model, we need to check, once more, that this model that we assumed is appropriate for our data. For this purpose, we carry out again a Monte Carlo test of goodness of fit (Besag and Diggle, 1977; Diggle, 1983).

We simulate 99 realizations of the model with the parameters estimated. One of these simulations can be seen in Fig. 8d. In order to describe the realizations we have chosen a generalization of the Ripley's $K$-function for second-order intensityreweighted stationary germ-grain models (Gallego et 
al., 2014).

$$
K_{\text {inhom }}(t)=\frac{1}{|W|} E\left(\int_{\Xi \cap W} \int_{\Xi \cap B(y, t)} \frac{1}{p(x) p(y)} \mathrm{d} y \mathrm{~d} x\right) .
$$

Let $\hat{K}_{\text {inhom }_{0}}$ and $\hat{K}_{\text {inhom }_{i}}(i=1, \cdots, 99)$ be the estimates of the $K_{\text {inhom }}$-function from the real image and the simulations. Fig. 9 shows the function estimated from the real data, $\hat{K}_{\text {inhom }_{0}}$, and the lower and upper envelopes of $\hat{K}_{\text {inhom }_{i}}$ estimated from the simulations. This indicates a satisfactory fit because $\hat{K}_{\text {inhom }}$ lies between the two envelopes except for a few values.

Monte Carlo method is applied, getting a p-value of 0.68 , which leads us to accept the null hypothesis formulated about the random distribution of callose deposition on leaves.

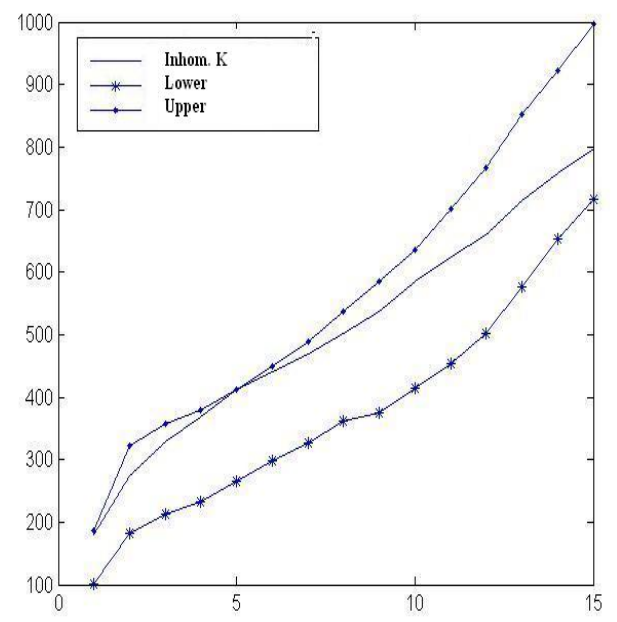

Fig. 9. Lower and upper envelopes of $\hat{K}_{\text {inhom }}$ estimates from the simulations and the estimated from the real data.

\section{CONCLUSIONS}

In this paper we have proposed a simple statistical method that can be used to estimate the parameters of a particular kind of non-homogeneous Boolean model. In this model a particular functional form for the intensity function of the underlying process can be assumed. The method is based on applying least squared fitting to the area fraction function and the density of the boundary length. We have shown that it provides estimators as accurate as those obtained with other more complex methods for the general case. As an illustration, this model and the method have been used to analyze microscopical images from a phytopathological application. They can be used to analyze images with similar characteristics in other scientific fields.

\section{ACKNOWLEDGMENTS}

We would like to thank Victor Flors from CAMN Department of the UJI for introducing us in this interesting problem and providing us the images. This work was supported by the Spanish Ministry of Economy and Competitiveness, project DPI201347279-C2-1-R and the Fundació Caixa Castelló BANCAIXA, project P1.1A2011.11.

\section{REFERENCES}

Ayala G (1988). Inferencia en modelos Booleanos. Ph.D. thesis, Department of Statistics. University of Valencia.

Baddeley A, Turner R (2005). Spatstat: an R package for analyzing spatial point patterns. J Stat Softw 12:1-42.

Baddeley AJ, Møller J, Waagepetersen R (2000). Nonand semi-parametric estimation of interaction in inhomogeneous point patterns. Stat Neerl 54:329-50.

Berman M, Turner T (1992). Approximating point process likelihoods with GLIM. J Roy Stat Soc C App 41:31-8.

Besag J, Diggle P (1977). Simple Monte Carlo tests for spatial pattern. J Roy Stat Soc C App 26:327-33.

Bond J, Kofman L, Pogosyan D (1995). How filaments of galaxies are woven into the cosmic web. Nature 380:603-6.

Bowman A, Azzalini A (1997). Applied smoothing techniques for data analysis: the kernel approach with S-Plus illustrations. Oxford University Press.

Brodatzki U, Mecke K (2001). Morphological model for colloidal suspensions. arXiv:cond.mat/0112009.

Chiu S, Stoyan D, Kendall W, Mecke J (2013). Stochastic Geometry and its applications. 3rd Ed. John Wiley \& Sons.

Cressie N (1993). Statistics for spatial data. Wiley Series in Probability and mathematical statistics. New York: Wiley-Interscience.

Diggle P (1983). Statistical analysis of spatial point patterns. London: Academic Press.

Fletcher R, Powell M (1963). A rapidly convergent descent method for minimization. Comput J 6:163-8.

Gallego M, Ibánez M, Simó A (2014). Inhomogeneous kfunction for germ-grain models. arXiv:1401.8115 .

Gill P, Murray W, Wright M (1981). Practical Optimization. Academic Press.

Goldfarb D (1970). A family of variable metric updates derived by variational means. Math Comp 24:23-6.

Han SP (1977). A globally convergent method for nonlinear programming. J Optimiz Theory App 22:297-309. 
Hahn U, Micheletti A, Pohlink R, Stoyan D, Wendrock H (1999). Stereological analysis and modelling of gradient structures. J Microsc 195:113-24.

Luna E, Pastor V, Robert J, Flors V, Mauch-Mani B, Ton J (2011). Callose deposition: A multifaceted plant defense response. Mol Plant Microbe In 24:183-93.

Lyman T (1972). Metals Handbook. American Society for Metals.

Margalef R (1974). Ecología. Barcelona: Omega.

Molchanov I (1997). Statistics of the Boolean model for practitioners and mathematicians. New York: J Wiley \& Sons.

Molchanov I, Chiu S (2000). Smoothing techniques and estimation methods for nonstationary Boolean models with applications to coverage processes. Biometrika 87:265-83.

Molchanov I, Stoyan D (1994). Asymptotic properties of estimators for parameters of the Boolean model. Adv Appl Probab 26:301-23.

Perry G, Enright BMN (2006). A comparison of methods for the statistical analysis of spatial point patterns in plant ecology. Plant Ecol 187:59-82.

Platt W, Evans G, Rathbun S (1988). The population dynamics of a long-lived conifer (Pinus palustris). Am Nat 131:491-525.
Plaza M (1991). Contrastes en modelos germen y grano. Ph.D. thesis, University of Valencia.

Powell MJD (1978a). The convergence of variable metric methods for nonlinearly constrained optimization calculations. In: Mangasarian OL, Meyer RR, Robinson SM, eds., Nonlinear Programming 3. Academic Press.

Powell MJD (1978b). A fast algorithm for nonlinearly constrained optimization calculations. In: Watson GA, ed. Numerical Analysis. Lect Not Math 630:144-57.

Quintanilla J, Torquato S (1997). Microstructure functions for a model of statistically inhomogeneous random media. Phys Rev E 55:1558-65.

Rathbun S, Cressie N (1994). A space-time survival point process for a longleaf pine forest in southern Georgia. $\mathrm{J}$ Am Stat Assoc 89:1164-74.

Schmitt M (1996). Estimation of intensity and shape in a non-stationary Boolean model. In: Jeulin D, ed. Advances in theory and applications of random sets. Proc Int Symp.

Schneider R, Weil W (2008). Stochastic and integral geometry. Probability and its applications. Heidelberg: Springer-Verlag.

Serra J (1982). Image analysis and mathematical morphology. London: Academic Press. pp. 481-502.

Weil W (2001). Densities of mixed volumes for Boolean models. Adv Appl Probab 33:39-60. 